\title{
Orexin A-induced enhancement of attentional processing in rats: role of basal forebrain neurons
}

Kristin N. Zajo

Coll William \& Mary, Dept Psychol, Williamsburg, VA 23187 USA

Joshua A. Burk

Coll William \& Mary, Dept Psychol, Williamsburg, VA 23187 USA

Jim R. Fadel

Univ S Carolina, Sch Med, Dept Pharmacol Physiol \& Neurosci, Columbia, SC 29208 USA

Follow this and additional works at: https://scholarworks.wm.edu/aspubs

\section{Recommended Citation}

Zajo, K. N., Fadel, J. R., \& Burk, J. A. (2016). Orexin A-induced enhancement of attentional processing in rats: role of basal forebrain neurons. Psychopharmacology, 233(4), 639-647.

This Article is brought to you for free and open access by the Arts and Sciences at W\&M ScholarWorks. It has been accepted for inclusion in Arts \& Sciences Articles by an authorized administrator of W\&M ScholarWorks. For more information, please contact scholarworks@wm.edu. 


\title{
Orexin A-induced enhancement of attentional processing in rats: role of basal forebrain neurons
}

\author{
Kristin N. Zajo ${ }^{1}$ Jim R. Fadel $^{2}$ • Joshua A. Burk ${ }^{1}$
}

Received: 27 July 2015 / Accepted: 26 October 2015 / Published online: 4 November 2015

(C) Springer-Verlag Berlin Heidelberg 2015

\begin{abstract}
Rationale Orexins are neuropeptides released in multiple brain regions from neurons that originate within the lateral hypothalamus and contiguous perfornical area. The basal forebrain, a structure implicated in attentional processing, receives orexinergic inputs. Our previous work demonstrated that administration of an orexin-1 receptor antagonist, SB-334867, systemically or via infusion directly into the basal forebrain, can disrupt performance in a task that places explicit demands on attentional processing.

Objectives Given that the orexin-1 receptor binds orexin A with high affinity, we tested whether orexin A could enhance attention in rats.

Methods Attentional performance was assessed using a task that required discrimination of variable duration visual signals from trials when no signal was presented. We also tested whether infusions of orexin A into the lateral ventricle could attenuate deficits following lesions of medial prefrontal cortical cholinergic projections that arise from the basal forebrain. Results Infusions of orexin A into the basal forebrain attenuated distracter-induced decreases in attentional performance. Orexin A attenuated deficits in lesioned animals when a visual distracter was presented.

Conclusion The present results support the view that orexin A can enhance attentional performance via actions in the basal
\end{abstract}

Joshua A. Burk

jabur2@wm.edu

1 Department of Psychology, College of William \& Mary, Williamsburg, VA 23187, USA

2 Department of Pharmacology, Physiology and Neuroscience, University of South Carolina School of Medicine, Columbia, SC 29208, USA forebrain and may be beneficial for some conditions characterized by attentional dysfunction due to disruption of cortical cholinergic inputs.

Keywords Acetylcholine - Attention · Basal forebrain · Hypocretin · Vigilance

\section{Introduction}

Since the initial reports of the discovery of orexins (de Lecea et al. 1998; Sakurai et al. 1998), these neuropeptides have been associated with numerous processes, including sleep, hunger, and drug abuse (Mahler et al. 2012; Ritchie et al. 2010; Sakurai et al. 1998; Thannickal et al. 2000). The role of orexins in attentional processing has also been investigated, with emphasis on the projections to the basal forebrain (Arrigoni et al. 2010; Fadel and Burk 2010). Loss of orexin neurons disrupts acquisition of conditioned orienting responses (Wheeler et al. 2014). Administration of an orexin-1 receptor antagonist, SB-334867, systemically or directly into the basal forebrain disrupts attentional performance (Boschen et al. 2009). These findings have supported the idea that orexins may be useful for treating cognitive deficits associated with the disruption of cortical cholinergic projections, such as in Alzheimer's disease (Fadel et al. 2013; Fronczek et al. 2012; Slats et al. 2012; Wennstrom et al. 2012).

The orexin-1 receptor has a much higher affinity for binding orexin A compared with orexin B (Sakurai et al. 1998). Intranasal or intravenous administration of orexin A can attenuate cognitive deficits following sleep deprivation in nonhuman primates (Deadwyler et al. 2007). Additionally, orexin A is thought to play an important role in different forms of learning and memory (Akbari et al. 2006, 2007; Aou et al. 2003; Jaeger et al. 2002; Piantadosi et al. 2015; Yang et al. 2013; but 
see also Dietrich and Jenck 2010). Although the evidence is somewhat mixed, overall, it appears that orexin A can reverse cognitive deficits under some conditions. However, the neural mechanisms for these effects of orexin A remain unclear. Basal forebrain corticopetal cholinergic neurons are known to be necessary for attentional performance. For example, immunotoxic lesions of these neurons disrupt signal detection in several attention-demanding tasks (McGaughy et al. 1996, 2002). Moreover, cortical acetylcholine release is increased during attention-demanding tasks (Arnold et al. 2002; Passetti et al. 2000). Using choline-sensitive biosensors, prefrontal cortical cholinergic transients are larger on trials with accurate signal detection following a previous trial when a response associated with a nonsignal was made (Howe et al. 2013). However, it is unknown whether the basal forebrain is part of the neural circuitry that mediates any attentionenhancing effects of orexin A. Moreover, the ability of orexin A to attenuate attentional deficits associated with damage to corticopetal cholinergic neurons is not well-studied.

In the present experiments, rats were trained in a two-lever task that required discrimination of brief, variable visual signals from trials when no signal was presented (Bushnell et al. 1994; McGaughy and Sarter 1995). This task was selected because of the sensitivity of the task to manipulations of basal forebrain corticopetal cholinergic neurons (e.g., McGaughy et al. 1996, 1999) and because of recent reports that demonstrated the translational potential and use of this task in multiple species, including mice (St. Peters et al. 2011) and humans (Bushnell et al. 2003; Demeter et al. 2008; Howe et al. 2013). The effects of infusions of orexin A directly into the basal forebrain were examined in a well-trained version of the task and with attentional demands increased by presenting a visual distracter. Given that orexins innervate multiple regions and the clinical interest associated with these neuropeptides, in a subsequent experiment, orexin A was infused into the lateral ventricle prior to attention task performance following loss of medial prefrontal cortical cholinergic inputs.

\section{Materials and methods}

\section{Subjects}

Male FBNF1 hybrid rats, $151-175 \mathrm{~g}$ at the beginning of the experiment were used (National Institute of Aging Colony). The rats were individually housed in a vivarium which was temperature and humidity controlled and operated on a 14:10 h light/dark cycle (lights on 0600). All rats were water restricted throughout the experiment, receiving water during behavioral testing and for $30 \mathrm{~min}$ after each testing session. Rats were trained 5 to 7 days a week, and received at least $1 \mathrm{~h}$ of water access on days when no behavioral testing occurred. Food was provided ad libitum throughout the experiment.
Animals were treated in accordance with the guidelines of the Animal Care and Use Committee at the College of William \& Mary.

\section{Apparatus}

Rats were trained in one of the 12 chambers, each located within a sound attenuating box (Med Associates, Inc.). Each chamber contained a water port positioned with a dipper that could be raised to provide water access $(0.01-\mathrm{ml}$ tap water). Two retractable levers were located on either side of the water port. A panel light was located above each lever and above the water port. A house light was positioned on the opposite side of each chamber. Illumination levels of these chambers have been described (Burk 2004). Behavioral testing programs and data collection were controlled by a personal computer using the Med-PC version IV software.

\section{Presurgical attention task training}

Training occurred between 9:00 a.m. and 12:00 p.m. daily. In the initial shaping procedure, rats were trained to press an extended lever using an FR-1 schedule of reinforcement, with the rule, to prevent a side bias, that if one lever was pressed five consecutive times, the other lever had to be pressed to receive water access. Once animals received 120 rewards in a session for three sessions, they were moved to the next training stage, where there were two trial types, signal (1-s illumination of the central panel light) and nonsignal (no illumination of the central panel light) trials. One second following a signal or no signal, the rats were cued to respond by extension of the levers into the chamber. Rules for training were counterbalanced, such that half of the rats were reinforced (3-s access to $0.01-\mathrm{ml}$ tap water) for pressing the left lever following a signal, which was recorded as a hit. A miss was recorded for right lever presses after a signal. For nonsignal trials, a press of the right lever was considered a correct rejection and water access was provided, while a press of the left lever on these trials was recorded as a false alarm. The rules of the task were reversed for the other half of the rats such that a right lever press was considered a hit following a signal presentation whereas the left lever was considered a correct response on a nonsignal trial. The inter-trial interval (ITI) was $12 \mathrm{~s}$, and the houselight was illuminated throughout the session during this stage of training. An incorrect response during this training phase would be followed by a correction trial which was the same trial type as that in which the error occurred. If the rat responded incorrectly for three consecutive trials, a forced trial occurred in which only the correct lever was extended into the chamber for $90 \mathrm{~s}$ or until the rat responded. If the errors occurred on signal trials, the central panel light was illuminated while the lever was extended. Each session lasted for $45 \mathrm{~min}$, and rats were trained with this 
task until reaching a criterion of $>70 \%$ accuracy on signal and nonsignal trials for three consecutive sessions.

After reaching criterion, rats were moved to the final stage of training prior to surgery. In the final task, the signal durations $(500,100,25 \mathrm{~ms})$ and ITI $(9 \pm 3 \mathrm{~s})$ were shorter and varied in order to increase explicit attentional demands (Parasuraman et al. 1987; Koelega et al. 1990). Each training session was comprised of 162 total trials (81 signal trials, 81 nonsignal trials). For the signal trials, each of the three signal durations was presented for 27 trials within a session. Trials were presented in blocks of 18 ( 9 nonsignal and 9 signal trials, with 3 of each signal duration), and trial types were selected randomly without replacement. Rats were considered trained for surgery when a criterion of $>70 \%$ accuracy on trials when the 500-ms signal was presented and on nonsignal trials for three consecutive sessions.

\section{Surgical procedures}

On the night prior to surgery, rats were provided with $2.7 \mathrm{mg} / \mathrm{ml}$ acetaminophen in their drinking water. Rats were anesthetized via IP injections of $90.0 \mathrm{mg} / \mathrm{kg}$ ketamine combined with $9.0 \mathrm{mg} / \mathrm{kg}$ xylazine. Once the rats were sufficiently anesthetized, the surgical area was shaved and rats were positioned in a stereotaxic device with the incisor bar set at $3.3 \mathrm{~mm}$ below the interaural line. All surgical procedures were conducted under aseptic conditions. In one experiment, rats received bilateral implantation of guide cannulae into the basal forebrain. For these rats, $8-\mathrm{mm}$ guide cannulae (22 gauge) were implanted at $-1.3 \mathrm{~mm}$ anterior-posterior (AP) and $\pm 2.7 \mathrm{~mm}$ medial-lateral (ML) from bregma and $-4.2 \mathrm{~mm}$ from dura. In a second experiment, rats received infusions of 192 IgG-saporin $(0.2 \mu \mathrm{g} / \mu \mathrm{l} ; 0.5 \mu \mathrm{l}$ per site) or saline into the medial prefrontal cortex ( 3.7 and $2.6 \mathrm{~mm} \mathrm{AP,} \pm 0.7 \mathrm{~mm} \mathrm{ML} ;-3.5 \mathrm{~mm}$ dorsal-ventral (DV); AP and ML from bregma, DV from dura) and a single guide cannula (hemisphere was randomized across rats) to allow infusions into the lateral ventricle $(-0.8 \mathrm{~mm} \mathrm{AP}$, $\pm 1.6 \mathrm{~mm}$ ML from bregma; $-2.5 \mathrm{~mm}$ DV from dura). Three stainless steel screws and dental cement were also used to secure the cannulae. For rats that received infusions into the medial prefrontal cortex, bone wax was applied above the skull holes to prevent the dental cement from entering these holes. Dummy cannulae were inserted to prevent blockage within the guide cannulae. Following surgery, animals were given a 1week recovery period in which food and water were available ad libitum. Rats were then returned to water restriction and began to retrain on the attention task.

\section{Postsurgical behavioral testing procedures prior to orexin A administration}

Rats that received bilateral basal forebrain cannulation were retrained in the same attention task as before surgery. After re- establishing criterion performance, rats were exposed to two sham infusion sessions, in which a short $(5-\mathrm{mm})$ internal cannula, attached to tubing, was inserted. The rats were then exposed to a form of the attention task where the houselight was flashed (1-s on/1-s off) during the middle block of trials within a testing session (trials 55-108).

Rats that received lateral guide cannula implantation and infusions of $192 \mathrm{IgG}$-saporin or saline into the medial prefrontal cortex were trained in the same attention task as before surgery for 15 sessions to establish any baseline effects of the lesion. Based upon previous findings, no effects of the lesion were expected on baseline task performance (Newman and McGaughy 2008). Rats were then exposed to two sham infusion sessions with the flashing houselight distracter during the middle block of trials, similar to rats with bilateral basal forebrain cannulae implantation. After these sham infusion sessions, all rats were considered ready for orexin A infusions.

\section{Drug administration procedures}

Infusions were made through the insertion of an internal cannula attached to a Hamilton syringe by polyethylene tubing. A total volume of $0.5 \mu \mathrm{l}$ solution was infused into each cannula at a rate of $1.0 \mu \mathrm{l} / \mathrm{min}$. The internal cannula was left in place for 1 min following the completion of each infusion to allow for drug diffusion. Animals were then immediately loaded into the chambers to begin behavioral testing. At least 1 day of training was allowed between each testing session to reestablish baseline performance. Orexin A (Tocris, Inc.) was dissolved in saline and aliquoted into small vials that were stored at $-20^{\circ}$ until being used for an infusion. Each vial was used only once on an infusion day and thus, orexin A was not repeatedly thawed and frozen. Rats with bilateral basal forebrain implantation received 0.0 (saline), 0.1, 1.0, and $10 \mathrm{pM}$ orexin A (Fadel et al. 2005) via internal cannulae (28 gauge) that extended $3.0 \mathrm{~mm}$ beyond the guide cannulae, with each dose being administered one time prior to task performance in an order that was randomized for each rat. The infusion procedures for rats receiving intracerebroventricular infusions were similar, except that the orexin A doses were increased $(0,10,100,1000 \mathrm{pM})$ via an internal cannula that extended $1.0 \mathrm{~mm}$ beyond the guide cannula. On drug infusion sessions, rats were tested in a version of the attention task with the flashing houselight distracter presented during the second block of trials (trials 55-108) within the session.

\section{Histological procedures and analysis}

After being deeply anesthetized via an IP injection of $100.0 \mathrm{mg} / \mathrm{kg}$ ketamine and $10.0 \mathrm{mg} / \mathrm{kg}$ xylazine, rats were transcardially perfused with $10 \%$ sucrose followed by $10 \%$ formalin (for rats with bilateral basal forebrain guide cannulae 
implanted) or $4 \%$ paraformaldehyde (for sham- or 192 IgGinduced lesions and lateral ventricle guide cannula implanted) at a pressure of $300 \mathrm{mmHg}$ using a Perfusion One apparatus. The brains were then removed and placed in the same fixative for $48 \mathrm{~h}$ before being put into a $30 \%$ sucrose solution in phosphate buffered saline for at least 3 days. The tissue was then sectioned in $50 \mu \mathrm{M}$ slices using a freezing microtome. For rats with bilateral guide cannulae in the basal forebrain, sections nearest the cannula sites were stained using cresyl violet and viewed under a microscope to assess cannula placement. For rats with cholinergic lesions or sham lesions, sections near the medial prefrontal cortex were stained for acetylcholinesterase (AChE)-positive fibers, and cannula location was also confirmed. AChE histochemistry was performed using a modification of previous procedures (Tago et al. 1986) and similar to previous work in our laboratory (Burk et al. 2008). Sections were first rinsed in a 0.1-M phosphate buffer solution and then incubated in hydrogen peroxide for $20 \mathrm{~min}$. After a rinse in maleate buffer, sections were immersed in a solution composed of $0.5 \mathrm{ml}$ of $0.1 \mathrm{M}$ sodium citrate, $1.0 \mathrm{ml}$ of $5 \mathrm{mM}$ potassium ferricyanide, $1.0 \mathrm{ml}$ of $30 \mathrm{mM}$ cupric sulfate, $10.0 \mathrm{mg}$ of acetylthiocholine, and $197.5 \mathrm{ml}$ of $0.1 \mathrm{M}$ maleate buffer for $45 \mathrm{~min}$. After rinsing in $50.0 \mathrm{mM}$ Tris buffer ( $\mathrm{pH} 7.6$ ), sections were incubated for $10 \mathrm{~min}$ in the solution prepared using the provided instructions in a diaminobenzidine (DAB) kit with nickel ammonium sulfate added. At the end of the incubation period, drops of $0.1 \%$ hydrogen peroxide were added to the sections until tissue staining was complete. These sections were mounted on gelatin-coated slides, dehydrated, and then cover slipped.

AChE-positive fibers were quantified in the prelimbic region of the medial prefrontal cortex as well as the primary motor (M1) regions in sections from both lesioned and sham-lesioned animals. AChE-positive fiber density was quantified using a modified counting grid method (Stichel and Singer 1987), similar to previous experiments in our laboratory and others (e.g., Burk et al. 2008; McGaughy et al. 1996; McGaughy and Newman 2008). Using an Olympus BX-51 light microscope with an objective lens magnification of $\times 40$, three lines that bisected each other at the midpoint were placed over the image using the Grid Mask function in ImagePro Discovery. Grid parameters were set to a radius of 400 with segments of 10 . Image size was set to $50 \%$. Each time a fiber crossed, the lines were counted in at least two sections in each hemisphere for all animals. The raw values were averaged together and used to estimate lesion extent.

\section{Behavioral measures and statistical analyses}

The number of hits $(H)$, misses $(M)$, correct rejections $(\mathrm{CR})$, false alarms (FA), and omissions were recorded for each animal during each testing session. The relative number of hits $[H /(H+M)]$ and false alarms $[\mathrm{FA} /(\mathrm{FA}+\mathrm{CR})]$ was calculated.
A sustained attention score (SAT) was then calculated to take into account accuracy on signal and nonsignal trials in which a lever press occurred. SAT was calculated using the following formula: $\mathrm{SAT}=\left((H-\mathrm{FA}) /\left(2 \times(H+\mathrm{FA})-(H+\mathrm{FA})^{2}\right)\right)($ Boschen et al. 2009; McGaughy et al. 1996). The range of scores for the SAT measure is from -1 to 1 , where a score of 1 indicates $100 \%$ correct responses on signal and nonsignal trials and a value of 0 indicates an inability to discriminate between signal and nonsignal trials. To assess accuracy separately on signal and nonsignal trials, the percentage of hits and correct rejections were calculated. During orexin A infusion sessions, the baseline version of the task, with the houselight consistently illuminated, occurred for the first 54 trials and then, for the next 54 trials, the houselight was flashed as a distracter. To determine the effect of the distracter for each rat, for each measure, the difference between block 1 (standard task with no distracter) and block 2 (distracter presentation) was calculated as a distracter score. Positive values indicate greater accuracy during block 1 and that the distracter decreased performance. Omissions were analyzed separately from measures of accuracy.

Data were analyzed with mixed factor ANOVAs, which included factors dose, lesion, and signal duration (where appropriate). Significant interactions were followed up by oneor two-way ANOVAs followed by $t$ tests. Data were analyzed with SPSS 19.0 for Windows (SPSS, Chicago, IL, USA). A level of $\alpha=0.05$ was used as the criterion for statistical significance and was adjusted using a modified Bonferroni procedure when multiple $t$ tests were conducted (Keppel 1991).

\section{Results}

\section{Effects of orexin A infused bilaterally into the basal forebrain}

A total of ten rats were included in this experiment. Only rats with appropriate cannulae placements (Fig. 1) and maintained criterion task performance between infusion sessions $(n=6)$ were included in these analyses. Intrabasalis orexin A did not affect performance on the standard task without the distracter (block 1). A dose $(0,0.1,1.0$, and $10 \mathrm{pM}$ orexin A) $\times$ signal duration $(500,100$, and $25 \mathrm{~ms})$ ANOVA for the SAT measure yielded no significant effects of orexin A during block 1. Similar analyses were conducted for the relative hits, as well as for nonsignal trials and omission rates. These analyses also yielded no significant effect of drug dose on any of these measures during the first block of trials.

Distracter scores were calculated for each rat for each behavioral measure to assess the effects of the distracter. This measure indicated the impairment in performance in response to the visual distracter presented during block 2 , with positive scores representing a more substantial decrease in attentional 

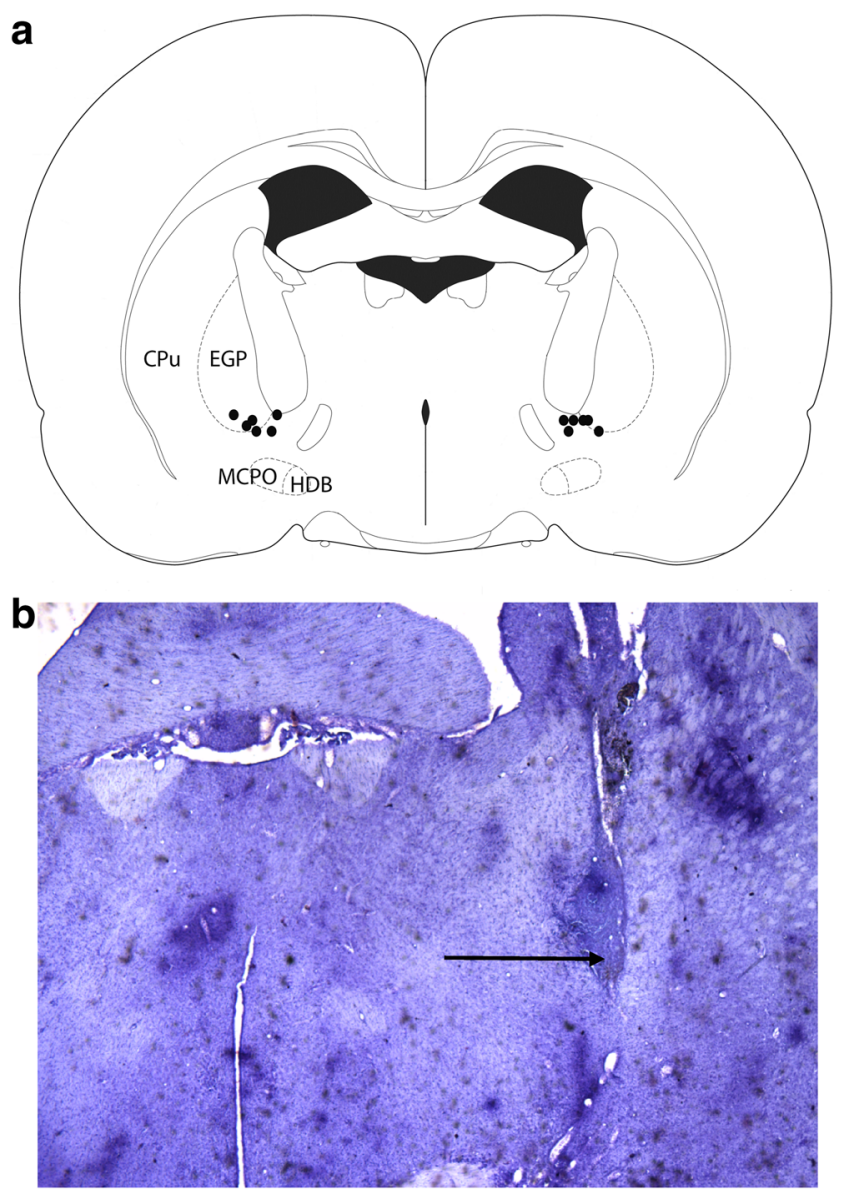

Fig. 1 The figure depicts the location of cannula placements for the six rats that were included in the analyses of attentional task performance (a). A photomicrograph, taken with a $\times 2$ objective, shows the cannula placement in one hemisphere of one animal, although all infusions were bilateral (b). The arrow indicates the infusion site. The section is approximately $-1.3 \mathrm{~mm}$ from bregma

performance when the distracter was presented. A dose $\times$ signal duration ANOVA did not yield main effects of either factor, but there was a significant dose $\times$ signal duration interaction $\left(F(6,30)=2.866, p<.05, \eta^{2}=.16\right)$ for the difference in SAT score measure. Follow-up analyses revealed a significant main effect of dose on trials when the 500-ms signal was presented $\left(F(3,15)=6.668, p<.05, \eta^{2}=.57\right)$, but not at the 100- or 25-ms signal durations (Fig. 2). This effect was mirrored by a dose $\times$ signal duration ANOVA for accuracy on signal trials. For hits, there was not a significant main effect of either factors, but the analysis did yield a significant dose $\times$ signal duration interaction for the distracter score $(F(6,30)=$ $\left.2.849, p<.05, \eta^{2}=.19\right)$. Separate one-way ANOVAs including dose as a factor were conducted for each signal duration. These analyses yielded a main effect of dose at the 500-ms signal duration $\left(F(3,15)=6.697, p<.05, \eta^{2}=.57\right)$, but not on the $100-$ or $25-\mathrm{ms}$ signal trials. Further analyses via paired sample $t$ tests compared each orexin A dose with vehicle administration. Performance following the 10-pM orexin A was
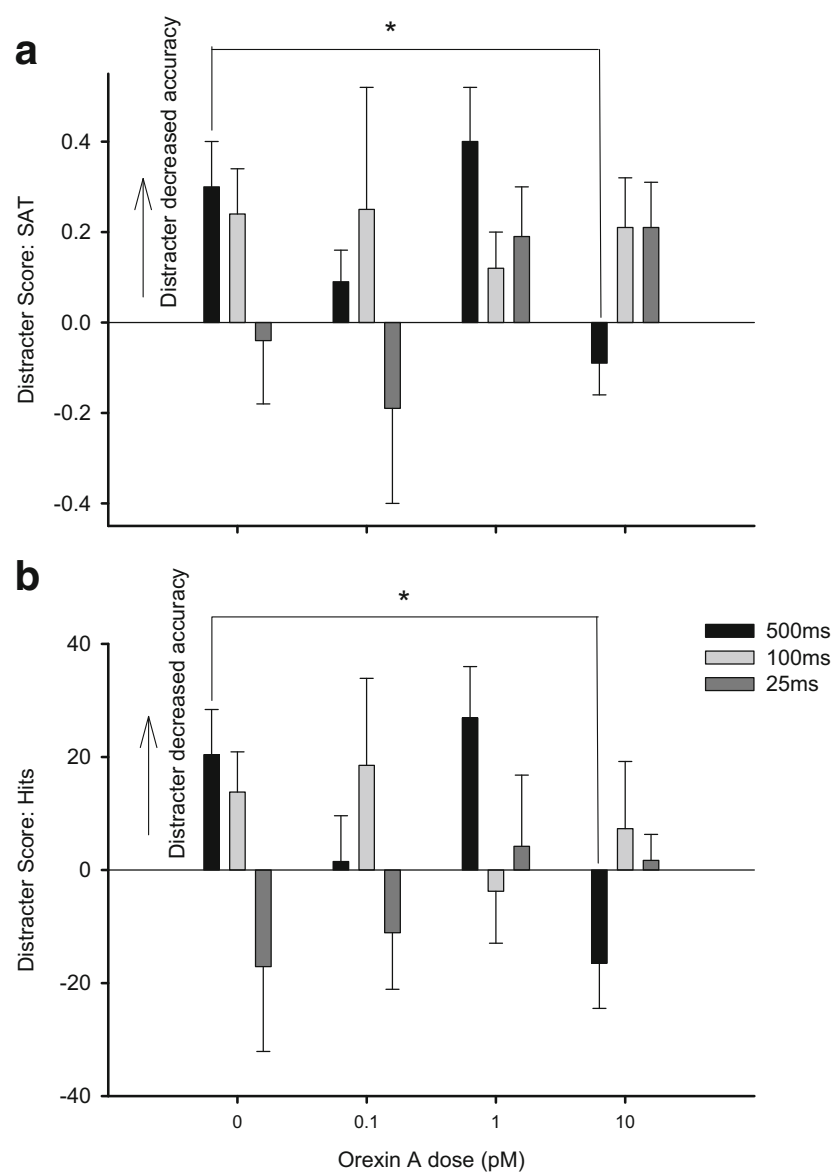

Fig. 2 The figure depicts the difference score (block 1-block 2) when the distracter was presented compared with the previous block of trials with no distracter for the SAT measure (a) and a similar difference score for percent hits (b). The $x$-axis depicts the orexin doses $(n=6)$

significantly different compared to vehicle at the longest (500 ms) signal duration $(t(5)=2.711, p<.05, d=1.11)$. Thus, at the longest $(500 \mathrm{~ms})$ signal duration, the $10-\mathrm{pM}$ orexin A dose decreased the distracter score for relative hits compared with vehicle administration. There were no significant effects of orexin A dose on correct rejections or omissions for the distracter score. Finally, dose $\times$ signal duration ANOVAs for the SAT measure, relative hits, correct rejections, and omissions on the standard task following the distracter (block 3) did not yield any significant results.

\section{Histological analyses of rats with medial prefrontal cortical infusions of 192 IgG-saporin or saline}

A total of 19 rats maintained stable performance throughout drug administration ( $n=9$ sham-lesioned rats and $n=10$ lesioned rats). On average, lesioned animals showed a $60.8 \%$ loss of AChE-positive fibers in the medial prefrontal cortex compared to sham-lesioned animals (Fig. 3). To assess whether the lesions produced a significant decrease in AChE fiber staining, $t$ tests comparing the mean AChE fiber counts from 

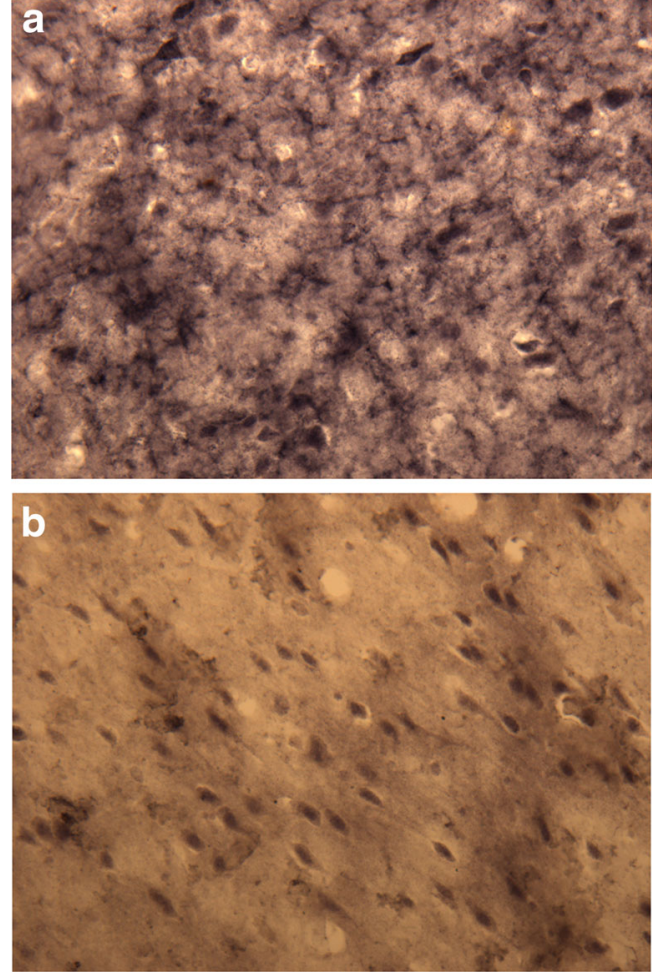

Fig. 3 The figure shows acetylcholinesterase fiber staining from the same lesioned animal, in primary motor cortex (a) or in the prelimbic region of the medial prefrontal cortex (b)

the medial PFC and M1 regions were conducted. This analysis confirmed that lesioned animals showed significantly fewer AChE fibers in the prelimbic region of the medial prefrontal cortex compared with sham-lesioned animals $(t(17)=10.769$, $p<.001, d=10.8$; mean \pm SEMs for AChE-positive fiber counts: sham-lesioned animals $61.18 \pm 3.61$, lesioned animals $23.98 \pm 1.62)$ but not in the primary motor cortex $(p>.79$; mean \pm SEMs for AChE-positive fiber counts: sham-lesioned animals $61.59 \pm 2.79$, lesioned animals $60.68 \pm 2.07$ ).

\section{Performance of lesioned or sham-lesioned rats prior to orexin A infusions}

No significant differences in measures of task performance were observed between lesioned and sham-lesioned groups on the 3 days prior to surgery. For the 15 sessions immediately after surgical recovery, performance was examined averaging measures from three consecutive sessions (e.g., average of sessions 1-3 and average of sessions 4-6). For relative hits, a repeated measures session (five levels) $\times$ signal duration (three levels) $\times$ lesion (two conditions) ANOVA did not yield any effects involving lesion. The lesioned animals did demonstrate a significant decrease in correct rejections compared to the sham-lesioned animals $(F(1,17)=5.962, p<.05$, $\eta^{2}=.26$ ), an effect that was significant during sessions $1-3$ $(t(17)=2.452, p<.05, d=1.0)$ and sessions 4-6 $(t(17)=2.223$, $p<.05, d=.93$ ), but not during subsequent testing sessions (all $p$ values $>.15$; Fig. 4 ). There were no effects involving lesion on omissions. Thus, immediately after surgery, lesioned rats demonstrated a transient decrease in correct rejections but the performance of this group was not significantly different from sham-lesioned animals after 15 postsurgical testing sessions.

\section{Effects of orexin A infusions on attention task performance of lesioned and sham-lesioned rats}

Following drug infusion, animals omitted nearly all trials following the highest orexin dose (1000 pM), rendering the measures of accuracy difficult to interpret for this dose. Therefore, measures of accuracy were analyzed only for sessions when either 0- (vehicle), 10-, or 100-pM orexin A was administered. For the SAT measure, a lesion $\times$ dose $(0,10,100 \mathrm{pM}$ orexin A) $\times$ signal duration ANOVA for the first block of trials yielded a significant lesion $\times$ dose $\times$ block interaction $(F(4$, $\left.64)=2.983, p<.05, \eta^{2}=.17\right)$. No other main effects or interactions involving lesion or dose were significant. The basis for this interaction appeared to be differences at the 25-ms signal duration; however, follow-up analyses did not reveal any statistically significant differences between lesioned and shamlesioned animals. A similar analysis was conducted for relative hits, correct rejections, and omissions during block 1 of trials and yielded no significant effects involving lesion or dose. The distracter score was calculated for each behavioral measure similar to those rats that received bilateral infusions of orexin A into the basal forebrain. For SAT, a lesion $\times$ dose $(0,10,100 \mathrm{pM}$ orexin $\mathrm{A}) \times$ signal duration ANOVA yielded a lesion $\times$ dose $\times$ signal duration interaction which was approaching significance $(F(4,64)=2.313, p=.067)$. There were

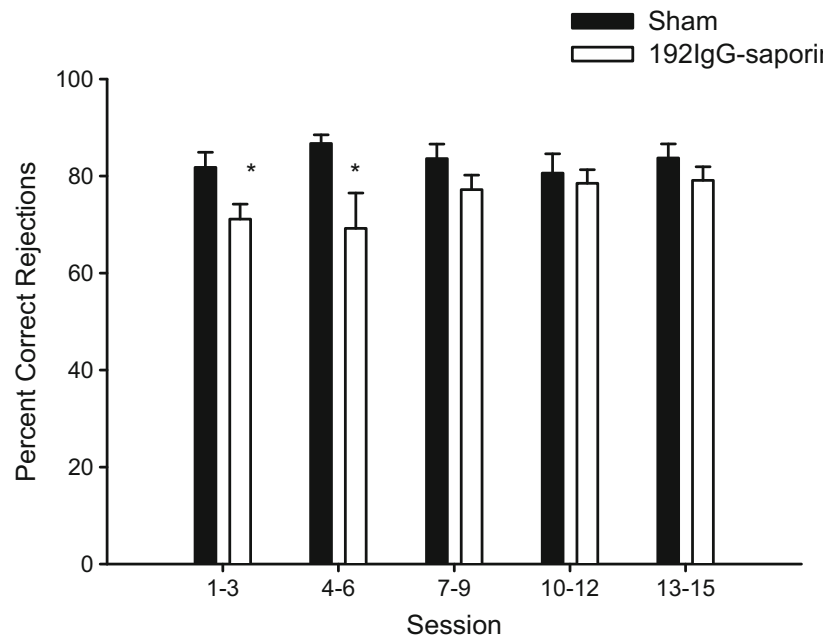

Fig. 4 The $x$-axis represents the average of the indicated sessions for sham-lesioned (darkened bars; $n=9$ ) and lesioned (open bars; $n=10$ ) animals. The $y$-axis depicts percent correct rejections 
no significant main effects or other interactions including lesion or dose. For relative hits, a lesion $\times$ dose $\times$ signal duration, revealed a significant lesion $\times$ dose $\times$ signal duration interaction $\left(F(4,68)=2.656, p<.05, \eta^{2}=.14\right)$ for the distracter score, but no main effects or other interactions involving lesion or dose. This interaction was further assessed by conducting separate dose $\times$ lesion ANOVAs at each signal duration. For relative hits, there was a significant dose $\times$ lesion interaction $(F(2$, $34)=4.965, p<.05, \eta^{2}=.21$ ), but no significant main effects, at the $25-\mathrm{ms}$ signal duration, but not on 500 - or $100-\mathrm{ms}$ signal trials. This dose $\times$ lesion interaction was further assessed with separate one-way ANOVAs for dose for sham-lesioned and lesioned animals on 25-ms signal trials. No significant effects of dose were observed for the sham-lesioned animals $(p>.50)$. There was, however, a significant main effect of dose on accuracy at the 25-ms signal duration for the lesioned group $\left(F(2,18)=6.922, p<.05, \eta^{2}=.43\right)$. To clarify the nature of this effect, paired sample $t$ tests comparing each orexin A dose with vehicle administration were conducted for the lesioned animals. These analyses yielded a significant difference in the distracter scores when vehicle administration was compared with $100 \mathrm{pM}$ orexin $\mathrm{A}(t(9)=2.973, p<.05, d=.94)$. Thus, at the 25-ms signal duration, the 100-pM orexin A dose decreased the distracter score for relative hits compared with vehicle administration, indicating that the lateral ventricle infusions of $100 \mathrm{pM}$ orexin A may reduce distractibility at the 25-ms level (Fig. 5). There were no significant effects of orexin A dose on correct rejections or omissions for the distracter score. Finally, lesion $\times$ dose $\times$ signal duration ANOVAs for the SAT measure and relative hits as well as lesion $\times$ dose ANOVAs for correct rejections and omissions on the standard task following the distracter (block 3) did not yield any significant effects involving dose or lesion.

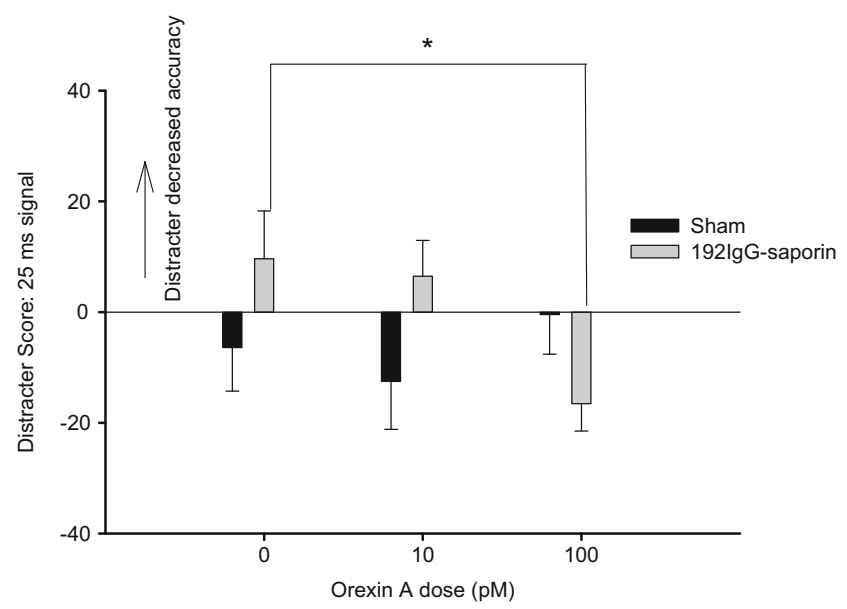

Fig. 5 The figure depicts the difference score (block 1-block 2) when the distracter was presented compared with the previous block of trials with no distracter for percent hits on trials when the $25-\mathrm{ms}$ signal was presented for sham-lesioned $(n=9)$ and for $192 \mathrm{IgG}$-saporin-induced lesioned animals $(n=10)$. The $x$-axis depicts the orexin doses

\section{Discussion}

The present experiments tested whether orexin A could enhance attentional performance via direct infusions into the basal forebrain and, in a separate experiment, via intraventricular infusion following sham- or 192 IgG-saporin-induced lesions of cholinergic projections to the medial prefrontal cortex. The present results extend previous findings by showing that attention-demanding aspects of task performance benefit the most from orexin A and by demonstrating that changes in attentional performance following loss of medial prefrontal cortex cholinergic inputs can be attenuated by orexin A.

In both experiments, beneficial effects of orexin A on attentional performance were observed when a visual distracter was presented. Thus, orexin A does not appear to enhance performance in a well-trained version of the task, but rather when attentional demands are augmented by a visual distracter. Moreover, the benefits of orexin A were not observed during the final block of trials, when subjects are recovering from the effects of the distracter. The orexin Ainduced performance enhancement specifically during the distracter condition suggests that the drug effects were not some general increase in arousal that improved all aspects of the task or specific facilitation of motivation. This conclusion is further supported by a lack of effect of orexin A on omissions.

Selective lesions of basal forebrain corticopetal cholinergic neurons typically decrease signal detection in this task (e.g., McGaughy et al. 1996, 1999). In the present study, the beneficial effects of orexin A were primarily observed on signal trials. These results potentially support the conclusion that the beneficial effects of orexin A are mediated via corticopetal cholinergic inputs. However, caution should be taken with this interpretation because orexins are also released onto basal forebrain noncholinergic neurons. The role of basal forebrain noncholinergic neurons in attention, and their interactions with cholinergic neurons, remains poorly understood (Sarter and Bruno 2002). Additionally, the obvious possibility exists that icv orexin A administration led to beneficial effects by actions outside of basal forebrain corticopetal neurons (Peyron et al. 1998). Lambe et al. (2005), for example, have demonstrated a pro-attentional effect of orexins mediated by orexin-2 receptors on thalamo-prefrontal projections.

When infused into the basal forebrain, orexin A increased the decline in performance on trials when the 500-ms signal was presented. Interestingly, this corresponds well with our previous work, showing that orexin-1 receptor blockade decreases accuracy on trials with a longer signal duration (Boschen et al. 2009). We previously suggested that this pattern of deficits reflected disrupted processing of the task rules for signal trials rather than signal perception. If rule processing is affected, then the change in performance would be predicted to occur following the longest signal, because this is the signal 
that the subject is most likely to detect. Collectively, we see that orexin receptor blockade and orexin A administration bidirectionally affect accuracy on 500-ms signal trials, although we did not find that orexin receptor blockade selectively affected accuracy on trials with a distracter (Boschen et al. 2009). The receptors responsible for the effects of orexin A require further investigation. Orexin A binds to both orexin1 and orexin-2 receptors (Sakurai et al. 1998), and these receptors are expressed at similar levels in the basal forebrain (Marcus et al. 2001). The present effects of orexin A could be due to actions at orexin- 1 or orexin- 2 receptors or activation of both of these receptor subtypes.

Lesions of the cholinergic projections to the medial prefrontal cortex transiently decreased correct rejections. It is somewhat surprising that the lesions affected accuracy on nonsignal, rather than signal trials, but the difference in performance supports that the lesions were effective. The extent of lesions was similar to previous studies in which this immunotoxin was infused into the medial prefrontal cortex (Newman and McGaughy 2008). As expected, the lesioned and sham-lesioned animals' performance did not differ during the final sessions prior to orexin A administration. Lesioned animals demonstrated improvement in detecting the $25-\mathrm{ms}$ signal following the 100-pM orexin A compared with vehicle administration when the distracter was presented. The 25-ms signal may be considered the most challenging to detect. Thus, the beneficial effects of orexin A for lesioned animals' performance are prominent during the highest attentional demands, both in terms of increased background noise and signal duration. The beneficial effects of intraventricular orexin $\mathrm{A}$ in lesioned animals does improve accuracy at a shorter signal duration compared to the effects of orexin A infused into the basal forebrain. It is certainly possible that, with these different methods of administration, in lesioned versus intact animals, orexin A is providing beneficial effects through different neural mechanisms. Further investigation of the neural basis of the beneficial effects of intraventricular orexin $\mathrm{A}$ is needed.

These results add to the growing literature about the beneficial cognitive effects of orexin A (Piantadosi et al. 2015). The basal forebrain appears to be one brain structure that mediates some of the effects of orexin A on attention. Collectively, these findings support the conclusion that orexin A is a fruitful target for future research into treatments for disorders characterized by attentional deficits.

Acknowledgments This research was supported by AG030646. The authors wish to thank Everett Altherr, Camille Hughes, John I. Kittle, Cassandra Leong, Jon MacLeod, Natalie McConnell, Nicole Mendoza, Diana Otoya, Cassidy Reich, Elizabeth Wolfe, and Petar Yanev for assistance with behavioral testing.

Compliance with ethical standards

Conflict of interest The authors declare no conflicts of interest.

\section{References}

Akbari E, Naghdi N, Motamedi F (2006) Functional inactivation of orexin 1 receptors in CA1 region impairs acquisition, consolidation and retrieval in Morris water maze task. Behav Brain Res 173:47-52

Akbari E, Naghdi N, Motamedi F (2007) The selective orexin 1 receptor antagonist SB-334867-A impairs acquisition and consolidation but not retrieval of spatial memory in Morris water maze. Peptides 28: 650-656

Aou S, Li XL, Li AJ, Oomura Y, Shiraishi T, Sasaki K, Imamura T, Wayner MJ (2003) Orexin-A (hypocretin-1) impairs Morris water maze performance and CA1-Schaffer collateral long-term potentiation in rats. Neuroscience 119:1221-1228

Arnold HM, Burk JA, Hodgson EM, Sarter M, Bruno JP (2002) Differential cortical acetylcholine release in rats performing a sustained attention task versus behavioral control tasks that do not explicitly tax attention. Neuroscience 114:451-460

Arrigoni E, Mochizuki T, Scammell TE (2010) Activation of the basal forebrain by the orexin/hypocretin neurones. Acta Physiol (Oxf) 198:223-235

Boschen KE, Fadel JR, Burk JA (2009) Systemic and intrabasalis administration of the orexin-1 receptor antagonist, SB-334867, disrupts attentional performance in rats. Psychopharmacology (Berlin) 206: 205-213

Burk JA (2004) Introduction of a retention interval in a sustained attention task in rats: effects of presenting a visual distracter and increasing the inter-trial interval. Behav Process 67:521-531

Burk JA, Lowder MW, Altemose KE (2008) Attentional demands for demonstrating deficits following intrabasalis infusions of 192 IgGsaporin. Behav Brain Res 22:231-238

Bushnell PJ, Kelly KL, Crofton KM (1994) Effects of toluene inhalation on detection of auditory signals in rats. Neurotoxicol Teratol 16: $149-160$

Bushnell PJ, Benignus VA, Case MW (2003) Signal detection behavior in humans and rats: a comparison with matched tasks. Behav Process 64:121-129

de Lecea L, Kilduff TS, Peyron C, Gao X, Foye PE, Danielson PE, Fukuhara C, Battenberg EL, Gautvik VT, Bartlett FS, Frankel WN, van del Pol AN, Bloom FE, Gautvik KM, Sutcliffe JG (1998) The hypocretins: hypothalamus-specific peptides with neuroexcitatory activity. Proc Natl Acad Sci U S A 95:322-3277

Deadwyler SA, Porrino L, Siegel JM, Hampson RE (2007) Systemic and nasal delivery of orexin-A (hypocretin-1) reduces the effects of sleep deprivation on cognitive performance in nonhuman primates. $\mathrm{J}$ Neurosci 27:14239-14247

Demeter E, Sarter M, Lustig C (2008) Rats and humans paying attention: cross-species task development for translational research. Neuropsychology 22:787-799

Dietrich H, Jenck F (2010) Intact learning and memory in rats following treatment with the dual orexin receptor antagonist almorexant. Psychopharmacology (Berlin) 212:145-154

Fadel J, Burk JA (2010) Orexin/hypocredtin modulation of the basal forebrain cholinergic system: role in attention. Brain Res 1314: $112-123$

Fadel J, Pasumarthi R, Reznikov LR (2005) Stimulation of cortical acetylcholine release by orexin A. Neuroscience 130:541-547

Fadel J, Jolivalt CG, Reagan LP (2013) Food for thought: the role of appetitive peptides in age-related cognitive decline. Ageing Res Rev 12:764-776

Fronczek R, van Geest S, Frolich M, Overeem S, Roelandse FW, Lammers GJ, Swaab DF (2012) Hypocretin (orexin) loss in Alzheimer's disease. Neurobiol Aging 33:1642-1650

Howe WM, Berry AS, Francois J, Gilmour G, Carp JM, Tricklebank M, Lustig C, Sarter M (2013) Prefrontal cholinergic mechanisms 
instigating shifts from monitoring for cues to cue-guided performance: converging electrochemical and fMRI evidence from rats and humans. J Neurosci 33:8742-8752

Jaeger LB, Farr SB, Banks WA, Morley JE (2002) Effects of orexin-A on memory processing. Peptides 23:1683-1688

Keppel G (1991) Design and analysis: a researcher's handbook, 3rd edn. Prentice Hall, Inc., Englewood Cliffs

Koelega HS, Brinkman JA, Zwep B, Verbaten MN (1990) Dynamic vs static stimuli and their effect on visual vigilance performance. Percept Mot Skills 70:823-831

Lambe EK, Olausson P, Horst NK, Taylor JR, Aghajanian GK (2005) Hypocretin and nicotine excite the same thalamocortical synapses in prefrontal cortex: correlation with improved attention in rat. $\mathrm{J}$ Neurosci 25:5225-5229

Mahler SV, Smith RJ, Moorman DE, Sartor GC, Aston-Jones G (2012) Multiple roles for orexin/hypocretin in addiction. Prog Brain Res 198:79-121

Marcus JN, Aschkenasi CJ, Lee CE, Chemilli RM, Saper CB, Yanagisawa M, Elmquist JK (2001) Differential expression of orexin receptors 1 and 2 in the rat brain. J Comp Neurol 435:6-25

McGaughy J, Sarter M (1995) Behavioral vigilance in rats: task validation and effects of age, amphetamine, and benzodiazepine receptor ligands. Psychopharmacology (Berlin) 117:340-357

McGaughy J, Kaiser T, Sarter M (1996) Behavioral vigilance following infusions of $192 \mathrm{IgG}$-saporin into the basal forebrain: selectivity of the behavioral impairment and relation to cortical AChE-positive fiber density. Behav Neurosci 110:247-265

McGaughy J, Decker MW, Sarter M (1999) Enhancement of sustained attention performance by the nicotinic acetylcholine receptor agonist ABT-418 in intact but not basal forebrain-lesioned rats. Psychopharmacology (Berlin) 144:175-182

McGaughy J, Dalley JW, Morrison CH, Everitt BJ, Robbins TW (2002) Selective behavioral and neurochemical effects of cholinergic lesions produced by intrabasalis infusions of $192 \mathrm{IgG}$-saporin on attentional performance in a five-choice serial reaction time task. J Neurosci 22:1905-1913

Newman LA, McGaughy J (2008) Cholinergic deafferentation of prefrontal cortex increases sensitivity to cross-modal distractors during a sustained attention task. J Neurosci 28:2642-2650

Parasuraman R, Warm JS, Dember WN (1987) Vigilance: taxonomy and utility. In: Mark LS, Warm JS, Huston RL (eds) Ergonomics and human factors. Springer, New York, pp 11-32

Passetti F, Dalley JW, O'Connell MT, Everitt BJ, Robbins TW (2000) Increased acetylcholine release in the rat medial prefrontal cortex during performance of a visual attentional task. Eur J Neurosci 12: 3051-3058
Peyron C, Tighe DK, van den Pol AN, de Lecea L, Heller HC, Sutcliffe JG, Kilduff TS (1998) Neurons containing hypocretin (orexin) project to multiple neuronal systems. J Neurosci 18:9996-10015

Piantadosi PT, Holmes A, Roberts BM, Bailey AM (2015) Orexin receptor activity in the basal forebrain alters performance on an olfactory discrimination task. Brain Res 1594:215-222

Ritchie C, Okuro M, Kanbayashi T, Nishino S (2010) Hypocretin ligand deficiency in narcolepsy: recent basic and clinical insights. Curr Neurol Neurosci Rep 10:180-189

Sakurai T, Amemiya A, Ishii M, Matsuzaki I, Chemelli RM, Tanaka H, Williams SC, Richarson JA, Kozlowski GP, Wilson S, Arch JR, Buckingham RE, Haynes AC, Carr SA, Annan RS, McNulty DE, Liu WS, Terrett JA, Elshourbagy NA, Bergsma DJ, Yanagisawa M (1998) Orexins and orexin receptors: a family of hypothalamic neuropeptides and $\mathrm{G}$ protein-coupled receptors that regulate feeding behavior. Cell 92:573-585

Sarter M, Bruno JP (2002) The neglected constituent of the basal forebrain corticopetal projection system: GABAergic projections. Eur J Neurosci 15:1867-1873

Slats D, Claassen JA, Lammers GJ, Melis RJ, Verbeek MM, Overseem S (2012) Association between hypocretin-1 and amyloid- $\beta 42$ cerebrospinal fluid levels in Alzheimer's disease and healthy controls. Curr Alzheimer Res 9:1119-1125

St Peters M, Cherian AK, Bradshaw M, Sarter M (2011) Sustained attention in mice: expanding the translational utility of the SAT by incorporating the Michigan Controlled Access Response Port (MICARP). Behav Brain Res 225:574-583

Stichel CC, Singer W (1987) Quantitative analysis of the choline acetyltransferase-immunoreactive axonal network in the cat primary visual cortex. J Comp Neurol 258:91-98

Tago H, Kimura H, Maeda T (1986) Visualization of detailed acetylcholinesterase fiber and neuron staining in rat brain by a sensitive histochemical procedure. J Histochem Cytochem 34:1431-1438

Thannickal TC, Moore RY, Nienhuis R, Ramanathan L, Gulyani S, Aldrich M, Cornford M, Siegel JM (2000) Reduced number of hypocretin neurons in human narcolepsy. Neuron 27:469-474

Wennstrom M, Londos E, Minthon L, Nielsen HM (2012) Altered CSF orexin and $\alpha$-synuclein levels in dementia patients. J Alzheimers Dis 29:125-132

Wheeler DS, Wan S, Miller A, Angeli N, Adileh B, Hu W, Holland PC (2014) Role of lateral hypothalamus in two aspects of attention in associative learning. Eur J Neurosci 40:2359-2377

Yang L, Zou B, Xiong X, Pascual C, Xie J, Malik A, Xie J, Sakurai T, Xie XS (2013) Hypocretin/orexin neurons contribute to hippocampusdependent social memory and synaptic plasticity in mice. J Neurosci 33:5275-5284 